\title{
SEIR EPIDEMIC MODEL WITH DELAY
}

\author{
PING YAN ${ }^{1}$ and SHENGQIANG LIU ${ }^{\bullet 2}$
}

(Received 5 October, 2005)

\begin{abstract}
A disease transmission model of SEIR type with exponential demographic structure is formulated, with a natural death rate constant and an excess death rate constant for infective individuals. The latent period is assumed to be constant, and the force of the infection is assumed to be of the standard form, namely, proportional to $I(t) / N(t)$ where $N(t)$ is the total (variable) population size and $I(t)$ is the size of the infective population. The infected individuals are assumed not to be able to give birth and when an individual is removed from the $I$-class, it recovers, acquiring permanent immunity with probability $f(0 \leq f \leq 1)$ and dies from the disease with probability $1-f$. The global attractiveness of the disease-free equilibrium, existence of the endemic equilibrium as well as the permanence criteria are investigated. Further, it is shown that for the special case of the model with zero latent period, $R_{0}>1$ leads to the global stability of the endemic equilibrium, which completely answers the conjecture proposed by Diekmann and Heesterbeek.
\end{abstract}

2000 Mathematics subject classification: primary 92D30; secondary 39B72.

Keywords and phrases: SEIR model, delay, conjecture, permanence, extinction, global stability.

\section{Introduction}

Mathematical models have become important tools in analysing the spread and control of infectious diseases. Attempts have been made to develop realistic mathematical models for the transmission dynamics of infectious diseases. The development of such models is aimed at both understanding observed epidemiological patterns and predicting the consequences of the introduction of public health interventions to control the spread of diseases. However, a model's ability to predict disease control depends greatly on the assumptions made in the modelling process [1]. Most epidemiological

\footnotetext{
'Department of Mathematics, University of Helsinki, FIN-00014, Finland; e-mail: ping.yan@helsinki.fi.

${ }^{2}$ Department of Mathematics, Xiamen University, Xiamen 361005, P. R. China; e-mail: sqliu@xmu.edu.cn.

(C) Australian Mathematical Society 2006, Serial-fee code 1446-181 1/06
} 
models descend from Kermack and McKendrick's classical SIR model in [20] (see, for example, $[3,11,14,16,19,25,26]$ and the references therein). Among them, Hethcote [14] proposed the following famous SIR model (see [14] and [15]) with vital dynamics (births and deaths) of:

$$
\begin{aligned}
& \frac{d S}{d t}=\mu N-\mu S-\gamma \frac{S I}{N}, \\
& \frac{d I}{d t}=-\mu I+\gamma \frac{S I}{N}-\alpha I, \\
& \frac{d R}{d t}=-\mu R+\alpha I,
\end{aligned}
$$

where the total population size $N(t)=S(t)+I(t)+R(t)$ and the per capita birth rate are assumed to be positive constants. Such assumptions, however, seem unrealistic because population size is always varying in the real world.

Later, in order to study disease within a variable population, Diekmann and Heesterbeek [9] improved system (1.1) by assuming that:

(1) The population has an exponential demographic structure.

(2) The infected individuals lose the ability to give birth.

(3) When an individual is removed from the $I$-class, he or she recovers and acquires permanent immunity with probability $f(0 \leq f \leq 1)$ and dies from the disease with probability $1-f$.

Thus Diekmann and Heesterbeek [9, page 56] modify model (1.1) into the following SIR model:

$$
\begin{aligned}
& \frac{d S}{d t}=b S+b R-\mu S-\gamma \frac{S I}{N}, \\
& \frac{d I}{d t}=-\mu I+\gamma \frac{S I}{N}-\alpha I, \\
& \frac{d R}{d t}=-\mu R+f \alpha I .
\end{aligned}
$$

Here $S$ denotes susceptible, $l$ infected and $R$ recovered individuals, $\mu$ is the per capita death rate due to causes other than the disease, $\gamma$ is the expected number of contacts per unit of time multiplied by the probability of transmission given contact, and $\alpha$ is the removal rate. The parameter $b$ is the per capita birth rate with $b>\mu$.

The assumptions of system (1.2) are more realistic than those for (1.1). However, in the natural world, for some diseases (for example, tuberculosis, influenza, measles) on adequate contact with an infective, a susceptible individual becomes exposed, that is, infected but not yet infective. This individual remains in the exposed class for a certain latent period before becoming infective (see, for example, Cooke et al. [8], Hethcote et al. $[17,18])$. Thus it is realistic for us to introduce a latent delay into 
system (1.2) and consider the corresponding SEIR epidemiologic model. We assume that the latent delay is constant, denoted by $\tau$. Using techniques similar to those in $[8,12,22]$, the probability that an individual survives the latent period $[t-\tau, t]$ is $e^{-\mu \tau}$, since the number of susceptible individuals that become exposed at time $t-\tau$ are $\gamma(S(t-\tau) I(t-\tau)) / N(t-\tau)$. There will then be

$$
\gamma \frac{S(t-\tau) I(t-\tau)}{N(t-\tau)} e^{-\mu \tau}
$$

individuals surviving in the latent period $\tau$ and becoming infective at time $t$. Thus we obtain the following delayed SEIR model:

$$
\begin{aligned}
& \frac{d S(t)}{d t}=b S(t)+b E(t)+b R(t)-\mu S(t)-\gamma \frac{S(t) I(t)}{N(t)}, \\
& \frac{d E(t)}{d t}=\gamma \frac{S(t) I(t)}{N(t)}-\gamma \frac{S(t-\tau) I(t-\tau)}{N(t-\tau)} e^{-\mu \tau}-\mu E(t), \\
& \frac{d I(t)}{d t}=-\mu I(t)+\gamma \frac{S(t-\tau) I(t-\tau)}{N(t-\tau)} e^{-\mu \tau}-\alpha I(t), \\
& \frac{d R(t)}{d t}=-\mu R(t)+f \alpha I(t),
\end{aligned}
$$

where $N(t)=S(t)+E(t)+I(t)+R(t)$ denotes the total population, $E$ denotes the number of exposed individuals and $\tau$ is the latent period. The other coefficients have the same definition as in model (1.2), with the following nonnegative initial conditions:

$$
S(t), E(t), I(t), R(t) \geq 0, \quad t \in[-\tau, 0], \quad N(t)>0 \text { on }[-\tau, 0] .
$$

For the continuity of the solutions to system (1.3), in this paper, we require

$$
E(0)=\int_{-\tau}^{0} \gamma \frac{S(u) I(u)}{N(u)} e^{\mu u} d u, \quad R(0)=0 .
$$

By system (1.3), we get

$$
\frac{d N(t)}{d t}=(b-\mu) N(t)-(b-(f-1) \alpha) I(t) .
$$

REMARK 1.1. It is easy to see that model (1.3) is an extended version of model (1.2) since (1.3) is reduced to (1.2) if $\tau=0$.

REMARK 1.2. Model (1.3) is different from some previous delay epidemiological models that engage non-delay coefficients $([2,3,25,29,30])$ in that only the infective term $I(t)$ of the incidence term $\gamma S(t) I(t) / N(t)$ has a delay $([2,3,25])$. 
REMARK 1.3. Model (1.3) is different from the SEIR model given by Cooke et al. [8]. In our model the infected individuals lose the ability to give birth, and when an individual is removed from the $I$-class, he or she recovers and acquires permanent immunity with probability $f(0 \leq f \leq 1)$ and dies from the disease with probability $1-f$.

By the second and the fourth equations of (1.3) and (1.5), we get

$$
E(t)=\int_{t-\tau}^{t} \gamma \frac{S(u) I(u)}{N(u)} e^{-\mu(t-u)} d u, \quad R(t)=\int_{0}^{t} f \alpha I(u) e^{-\mu(t-u)} d u .
$$

Define

$$
s(t)=\frac{S(t)}{N(t)}, \quad e(t)=\frac{E(t)}{N(t)}, \quad i(t)=\frac{I(t)}{N(t)}, \quad r(t)=\frac{R(t)}{N(t)},
$$

and $m(t)=b-(b+(1-f) \alpha) i(t)$, then (1.6) becomes

$$
\frac{d N(t)}{d t}=(m(t)-\mu) N(t)
$$

It follows from (1.5) and (1.7)-(1.9) that $N(t)=N(t-\tau) \exp \left(\int_{t-\tau}^{t} m(s) d s\right)$. System (1.3) becomes the following equivalent integro-differential equation system for $t>0$ :

$$
\begin{aligned}
\frac{d s(t)}{d t} & =b-b i(t)-m(t) s(t)-\gamma s(t) i(t), \\
\frac{d e(t)}{d t} & =\gamma s(t) i(t)-\gamma s(t-\tau) i(t-\tau) e^{-\int_{t-\mathrm{r}}^{t} m(s) d s}-m(t) e(t), \\
\frac{d i(t)}{d t} & =\gamma s(t-\tau) i(t-\tau) e^{-\int_{t-\mathrm{t}}^{t} m(s) d s}-(m(t)+\alpha) i(t), \\
\frac{d r(t)}{d t} & =f \alpha i(t)-m(t) r(t)
\end{aligned}
$$

with

$$
\begin{aligned}
& s(t), e(t), i(t), r(t) \geq 0, \quad t \in[-\tau, 0], \\
& s(t)+e(t)+i(t)+r(t)=1 \quad \text { on }[-\tau, 0]
\end{aligned}
$$

as the initial conditions.

By the second and the fourth equations of (1.10) and (1.5), we get

$$
\begin{aligned}
& e(t)=\int_{t-\tau}^{t} \gamma s(u) i(u) e^{-\int_{u}^{t} m(s) d s} d u, \\
& r(t)=\int_{0}^{t} f \alpha i(u) e^{-\int_{u}^{t} m(s) d s} u .
\end{aligned}
$$

For system (1.3), using arguments similar to those of [8, Corollary 2.1], we have the following result. 
LEMMA 1.1. Let $S(t), E(t), I(t), R(t)$ be the solution of system (1.3) on $t \geq 0$ with initial conditions (1.4). Then $s(t), e(t), i(t), r(t)$ is the solution of $(1.10)$ with initial conditions (1.11). Moreover, $s(t), e(t), i(t), r(t) \geq 0, t \geq 0$. If $s(t)$ and $i(t)$ are positive on the initial interval, then $s(t)$ and $i(t)$ are positive for all $t \geq 0$.

Epidemic models with delays have received much attention since delays can often cause some complicated dynamical behaviours. Delays in many population dynamics models can destabilise an equilibrium and thus lead to periodic solutions by Hopf bifurcation [21]. Similar results are also obtained for epidemiological models (Brauer et al. [4,5]; Busenberg et al. [6,7]; Hethcote et al. [15, 17, 19]). It is interesting for us to consider the effects of time delay on the dynamical behaviours of model (1.3).

In this paper, by constructing a proper Lyapunov function, we get the global stability of the disease-free proportion equilibrium. Using Thieme's persistence criteria [27] (for persistence and its application, and also referring to the works of Hale and Waltmann [13,28], Liu et al. [23, 24], and Xiao and Chen [31]), we get the delaydependent sufficient conditions under which the system is endemic in the sense of permanence. Our disease-free result generalises the corresponding results in Diekmann and Heesterbeek [9]. Further, we prove that for the special case of model (1.3) without latent period $\tau$, that is, system (1.2), existence of the endemic equilibrium indicates its global stability, which completely answers the conjecture proposed by Diekmann and Heesterbeek [9].

This paper is organised as follows. In Section 2, we obtain the threshold and the two equilibria of model (1.3). Stability of the disease-free equilibria are presented in Section 3. In Section 4, sufficient conditions for permanence of system (1.10) are obtained. In Section 5, we prove that for the special case of model (1.3) without a latent period $\tau$, existence of the endemic equilibrium indicates its global stability, which completely answers the conjecture proposed by Diekmann and Heesterbeek [9]. Finally, in Section 6 we summarise and discuss the results of this paper.

\section{Preliminary results}

Now we consider the equilibria of system (1.10). When the infective fraction $i=0$, then $e=r=0$, and $s=1$. This is the disease-free equilibrium for proportions. We note that this is the only equilibrium on the boundary of $D$. We have the following threshold parameter for the existence of interior equilibrium:

$$
R_{0}=\gamma e^{-b \tau} /(b+\alpha) .
$$

Denote $\left(s^{*}, e^{*}, i^{*}, r^{*}\right)$ as the interior equilibrium of (1.10). Since $s^{*}+e^{*}+i^{*}+r^{*}=1$, then $0<s^{*}, i^{*}<1$. By the first and third equations of (1.10), we get

$$
b-b i^{*}-m^{*} s^{*}-\gamma s^{*} i^{*}=0, \quad \gamma s^{*} i^{*} e^{-\int_{t-\varepsilon}^{*} m^{*} d s}-\left(m^{*}+\alpha\right) i^{*}=0,
$$


where $m^{*}=b-(b+(1-f) \alpha) i^{*}$. By the first and second equations of (2.2), we have

$$
\begin{aligned}
s^{*} & =\frac{b\left(1-i^{*}\right)}{b+[\gamma-b-(1-f) \alpha] i^{*}} \quad \text { and } \\
s^{*} & =\frac{(b+\alpha)-[b+(1-f) \alpha] i^{*}}{\gamma \exp (-b \tau) \exp \left\{[b+(1-f) \alpha] i^{*} \tau\right\}},
\end{aligned}
$$

and thus

$$
\frac{(b+\alpha)-[b+(1-f) \alpha] i^{*}}{\gamma \exp (-b \tau) \exp \left\{[b+(1-f) \alpha] i^{*} \tau\right\}}=\frac{b\left(1-i^{*}\right)}{b+[\gamma-b-(1-f) \alpha] i^{*}},
$$

that is,

$$
\frac{1}{R_{0}} \frac{1-[b+(1-f) \alpha] i^{*} /(b+\alpha)}{\exp \left\{[b+(1-f) \alpha] i^{*} \tau\right\}}=\frac{b\left(1-i^{*}\right)}{b+[\gamma-b-(1-f) \alpha] i^{*}} .
$$

Let $G\left(i^{*}\right)$ denote the difference between the left- and right-hand sides of (2.4). Thus we have $G(0)=1 / R_{0}-1$ and $G(1)>0$. Then if $R_{0}>1$, we have $G(0)<0$, which implies that Equation (2.4) admits at least one positive solution $i^{*} \in(0,1)$. Using (2.3) and (1.10), we can get the corresponding $s^{*}, e^{*}, r^{*}$. Therefore we get the following lemma.

LEMMA 2.1. System (1.10) has at least an endemic equilibrium if $R_{0}>1$.

\section{Disease-free equilibrium}

THEOREM 3.1. If $\gamma<b+\alpha$, all solutions of system (1.10) with initial conditions (1.4) will approach the disease-free equilibrium as $t \rightarrow \infty$.

PROOF. Let $s(t), e(t), i(t), r(t)$ be a solution of (1.10). We define

$$
V(t)=e(t)+i(t) .
$$

From (1.10) and noting $0 \leq s(t), e(t), i(t), r(t) \leq 1, s(t)+e(t)+i(t)+r(t)=1$, we have

$$
\begin{aligned}
\dot{V}(t)= & \gamma s(t) i(t)-m(t)(e(t)+i(t))+f \alpha i(t) \\
= & \gamma s(t) i(t)-(b-[b+(1-f) \alpha) i(t)](1-s(t)-r(t))+f \alpha i(t) \\
= & \{\gamma-[b+(1-f) \alpha]\} s(t) i(t)-[b+(1-f) \alpha] i(t) r(t)-f \alpha i(t)-b e(t) \\
= & \{\gamma-[b+(1-f) \alpha]-f \alpha\} s(t) i(t)-[b+(1-f) \alpha] i(t) r(t) \\
& -b e(t)-f \alpha i(t)(1-s(t))
\end{aligned}
$$




$$
=\{\gamma-[b+\alpha]\} s(t) i(t)-[b+(1-f) \alpha] i(t) r(t)-b e(t)-f \alpha i(t)(1-s(t)) \leq 0 .
$$

Then $\lim _{t \rightarrow \infty} V(t)$ exists and we have $\lim _{t \rightarrow \infty} V(t) \geq 0$. We prove $\lim _{t \rightarrow \infty} V(t)=0$. Assume that it is not true, that is, that $\lim _{t \rightarrow \infty} V(t)>0$. Thus

$$
\varliminf_{t \rightarrow \infty}(1-s(t)) \geq \lim _{t \rightarrow \infty} V(t)>0 .
$$

Hence we get

$$
\dot{V}(t) \leq-b e(t)-f \alpha i(t)(1-s(t)) \leq-\beta V(t) .
$$

Here $\beta=\min \left\{b, f \lim _{t \rightarrow \infty} V(t)\right\}$, which indicates that $\lim _{t \rightarrow \infty} V(t)=0$, a contradiction. This proves Theorem 3.1.

\section{Endemic equilibrium}

In this section, we prove that $R_{0}>1$ implies that system (1.10) is permanent. There have been many papers devoted to the persistence theory of delay differential equations (see Hale and Waltman [13], Freedman and Moson [10], Thieme [27, 28] and the references therein). In this paper, we engage Thieme's persistence theory [27]. Before stating our theorem, we present the following definitions that are similar to those in $[21,31]$.

DEFINITION 1. System (1.10) is said to be uniformly persistent if there is an $\eta>0$ (independent of the initial data) such that every solution $(s(t), e(t), i(t), r(t))$ with positive initial conditions satisfies:

$$
\lim _{t \rightarrow \infty} \inf s(t) \geq \eta, \quad \lim _{t \rightarrow \infty} \inf e(t) \geq \eta, \quad \lim _{t \rightarrow \infty} \inf i(t) \geq \eta, \quad \lim _{t \rightarrow \infty} \inf r(t) \geq \eta .
$$

DEFINITION 2. System (1.10) is said to be permanent if there exists a compact region $\Omega_{0} \subset$ Int $\Omega$ such that every solution of Equation (1.10) with positive initial conditions will eventually enter and remain in region $\Omega_{0}$.

Clearly, for a dissipative system, uniform persistence is equivalent to permanence.

THEOREM 4.1. System (1.10) is permanent provided that $R_{0}>1$.

To prove Theorem 4.1, we need the following lemma.

LEMMA 4.2 (Liu et al. [24]). Given $d^{*}>d$ and the system

$$
\begin{aligned}
& v^{\prime}(t)=d^{*} v(t-\tau)-d v(t), \quad v(t)=\theta(t) \geq 0, \quad t \in[-\tau, 0], \\
& v(0)>0,
\end{aligned}
$$

then $\lim _{t \rightarrow \infty} v(t)=+\infty$. 
And now we present the persistence criteria by Thieme [27]. Consider a metric space $X$ with metric $d$. Let $X$ be the union of two disjoint subsets $X_{1}, X_{2}$, and $\Phi$ a continuous semiflow on $X_{1}$, that is, a continuous mapping $\Phi:[0, \infty) \times X_{1} \rightarrow X_{1}$ with the following properties: $\Phi_{t} \circ \Phi_{s}=\Phi_{t+s}$, for $t, s \geq 0$, and $\Phi_{0}(x)=x$, for $x \in X_{1}$. Here $\Phi_{t}$ denotes the mapping from $X_{1}$ to $X_{1}$ given by $\Phi_{t}(x)=\Phi(t, x)$. The distance $d(x, Y)$ of a point $x \in X$ from a subset $Y$ of $X$ is defined by

$$
d(x, y)=\inf _{y \in Y} d(x, y) .
$$

Let $Y_{2}$ be a subset of $X_{2} ; Y_{2}$ is called a weak repeller for $X_{1}$ if, for all $x_{1} \in X_{1}$, limsup $\sup _{t \rightarrow \infty} d\left(\Phi_{1}\left(x_{1}\right), Y_{2}\right)>0$, and $Y_{2}$ is called a uniform strong repeller for $X_{1}$ if there is some $\epsilon>0$ such that $\liminf _{t \rightarrow \infty} d\left(\Phi_{t}\left(x_{1}\right), Y_{2}\right)>\epsilon$, for all $x_{1} \in X_{1}$.

$\left(\mathrm{H}_{1}\right)$ There exist $\delta>0$ and a subset $B$ of $X$ with the following properties:

- If $x \in X$ and $d\left(x, X_{2}\right)<\delta$, then $d\left(\Phi_{t}(x), B\right) \rightarrow 0, t \rightarrow \infty$.

- $B \bigcap B_{\delta} X_{2}$ has a compact closure. Here $B_{\delta} X_{2}=\left\{x \in X ; d\left(x, X_{2}\right)<\delta\right\}$.

LEMMA 4.3 ([27, Theorem 4.6]). Let $X_{1}$ be open in $X$ and forward invariant under $\Phi$. Further, let $\left(\mathrm{H}_{1}\right)$ hold. Assume that

$$
\Omega_{2}=\bigcup_{y \in Y_{2}} \omega(y), \quad Y_{2}=\left\{x \in X_{2} ; \Phi_{t}(x) \in X_{2}, \forall t>0\right\}
$$

where $\Omega_{2}$ has an acyclic isolated covering $M=\bigcup_{k=1}^{m} M_{k}$ such that each part $M_{k}$ of $M$ is a weak repeller for $X_{1}$. Then $X_{2}$ is a uniform strong repeller for $X_{1}$.

We are now able to prove Theorem 4.1.

PROOF OF THEOREM 4.1. We begin by considering the following subsystem (4.1) of system (1.10):

$$
\begin{aligned}
& \frac{d s(t)}{d t}=b-b i(t)-m(t) s(t)-\gamma s(t) i(t), \\
& \frac{d i(t)}{d t}=\gamma s(t-\tau) i(t-\tau) e^{-\int_{t-\tau}^{t} m(s) d s}-(m(t)+\alpha) i(t) .
\end{aligned}
$$

Claim 1. $R_{0}>1$ leads to the permanence of system (4.1).

By (1.8) and the initial conditions of system (1.10), we have that the initial conditions for (4.1) are

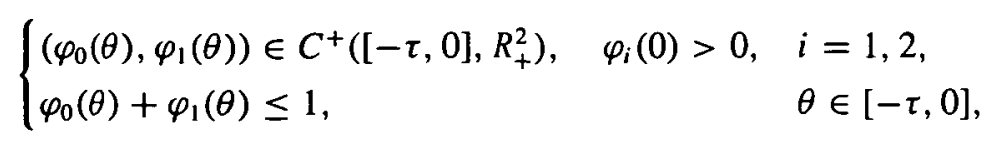


where $R_{+}^{2}=\left\{(s, i) \in R^{2}: s, i \geq 0 ; s+i \leq 1\right\}$. Let $C^{+}\left([-\tau, 0], R_{+}^{2}\right)$ denote the space of continuous functions mapping $[-\tau, 0]$ into $R_{+}^{2}$. We choose

$$
\begin{aligned}
& C_{1}=\left\{\left(\varphi_{0}, \varphi_{1}\right) \in C^{+}\left([-\tau, 0], R_{+}^{2}\right): \varphi_{0}(\theta) \equiv 0, \varphi_{1}(\theta)>0, \theta \in[-\tau, 0]\right\}, \\
& C_{2}=\left\{\left(\varphi_{0}, \varphi_{1}\right) \in C^{+}\left([-\tau, 0], R_{+}^{2}\right): \varphi_{0}(\theta) \geq 0, \varphi_{1}(\theta) \equiv 0, \theta \in[-\tau, 0]\right\} .
\end{aligned}
$$

Denote $X_{2}=C_{1} \cup C_{2}, X=C^{+}\left([-\tau, 0], R_{+}^{2}\right)$ and $X_{1}=\operatorname{Int} C^{+}\left([-\tau, 0], R_{+}^{2}\right)$, then $X_{2}=\partial X_{1}$.

We verify below that the conditions for Lemma 4.3 are satisfied.

The definitions of $X_{1}$ and $X_{2}$ imply that $X_{1}, X_{2}$ are disjoint sets and that $X_{1}$ is open. And by Lemma 1.1, we get $s(t), i(t), e(t), r(t)>0, t>0$. Thus $s(t)+i(t)<1$ for all $t>0$, proving that $X_{1}$ is positively invariant.

To show that condition $\left(\mathrm{H}_{1}\right)$ of Lemma 4.3 holds, select $B=X$ and an arbitrary positive constant $\delta$. We note that $X$ is also invariant. Then $d\left(\Phi_{t}(x), B\right)=$ $d\left(\Phi_{t}(x), X\right)=0, x \in X$. And $B \bigcap B_{\delta} X_{2}=X \bigcap B_{\delta} X_{2}=\left\{x \in X ; d\left(x, X_{2}\right)<\delta\right\}$. Hence the interaction of $B \bigcap B_{\delta} X_{2}$ has a compact closure $\left\{x \in X ; d\left(x, X_{2}\right) \leq \delta\right\}$.

Consider the $\Omega_{2}$ in Lemma 4.3. By system (4.1), all points in $C_{1}$ will ultimately enter $X_{1}$ while those in $C_{2}$ will converge to the constant solution $\widetilde{E}_{1}$ with

$$
\widetilde{E_{1}}=\left\{\left(\varphi_{0}, \varphi_{1}\right) \in C^{+}\left([-\tau, 0], R_{+}^{2}\right): \varphi_{0}(\theta) \equiv 1, \varphi_{1}(\theta) \equiv 0, \theta \in[-\tau, 0]\right\} .
$$

Hence $\Omega_{2}=\left\{\widetilde{E_{1}}\right\}$. Clearly it is isolated and acyclic.

Now we prove $\widetilde{E_{1}}$ is a weak repeller for $X_{1}$. Assume the contrary, that is, that there exists a positive solution $(s(t), i(t))$ of system (4.1) with $\lim _{t \rightarrow \infty}(s(t), i(t))=(1,0)$. Then for sufficiently small $\varepsilon$ with $\varepsilon<\left(1-(b+\alpha) e^{b \tau} / \gamma\right) / 2$, there exists a positive constant $T=T(\varepsilon)$ such that $s(t)>1-\varepsilon, 0<i(t)<\varepsilon$ for all $t \geq T$. Then we have $m(t)<b$ for all $t \geq T$. By the second equation of (4.1), we have

$$
\frac{d i(t)}{d t}>\gamma(1-\varepsilon) i(t-\tau) e^{-b \tau}-(b+\alpha) i(t), \quad t \geq T+\tau .
$$

Consider the equation

$$
\begin{cases}d x(t) / d t=\gamma(1-\varepsilon) x(t-\tau) e^{-b \tau}-(b+\alpha) x(t), & t \geq T+\tau, \\ x(t) \equiv i(t), & t \in[T, T+\tau] .\end{cases}
$$

By (4.2) and the comparing theorem, we have $i(t) \geq x(t)$ for all $t>T$. On the other hand,

$$
\begin{aligned}
\gamma(1-\varepsilon) e^{-b \tau}-(b+\alpha) & >\gamma\left(1-\frac{1}{2}\left(1-\frac{(b+\alpha) e^{b \tau}}{\gamma}\right)\right) e^{-b \tau}-(b+\alpha) \\
& =\frac{1}{2}\left(\gamma e^{-b \tau}-(b+\alpha)\right)=\frac{1}{2}(b+\alpha)\left(R_{0}-1\right)>0 .
\end{aligned}
$$


By Lemma 4.2, we get $x(t) \rightarrow \infty$ as $t \rightarrow \infty$. Thus $i(t) \rightarrow \infty$ as $t \rightarrow \infty$, contradicting $i(t)<\varepsilon,(t \geq T)$. Then system (4.1) satisfies all conditions of Lemma 4.3 and we have that $X_{2}$ is a uniformly strong repeller for $X_{1}$. Noting system (4.1) is dissipative, then system (4.1) is permanent. This proves Claim 1.

By (1.12), we can get the permanence of $e(t), r(t)$, thus proving Theorem 4.1.

\section{Model (1.3) with zero latent period}

In this section, we study system (1.2), that is, system (1.3) with $\tau=0$. By (2.1), noting $\tau=0$ in system (1.2), we get the basic reproduction number for system (1.2): $R_{0}=\gamma /(b+\alpha)$. Let

$$
R_{0}>1 \text {. }
$$

Introducing the relative quantities $y=I / N, z=R / N$ into (1.2) with $N=S+I+R$ being the total population, one obtains the two-dimensional system

$$
\begin{aligned}
& \dot{y}=y(\gamma(1-y-z)-\alpha+\alpha(1-f) y+b(y-1)) \equiv F_{1}(y, z), \\
& \dot{z}=y(f \alpha+(1-f) \alpha z)-b z(1-y) \equiv F_{2}(y, z),
\end{aligned}
$$

supplemented by the scalar equation

$$
\frac{d N}{d t}=(b-\mu-(b+\alpha(1-f)) y) N .
$$

Then the system (5.2) has a unique nontrivial equilibrium $(\bar{y}, \bar{z})(0<\bar{y}<1$, $0<\bar{z}<1,0<\bar{y}+\bar{z}<1$ ). For the endemic equilibrium, we have the following theorem.

THEOREM 5.1. If (5.1) is satisfied, then $(\bar{y}, \bar{z})$ is globally asymptotically stable in the set $D=\{(y, z): y>0, z>0, y+z \leq 1\}$.

REMARK 5.1. Theorem 5.1 gives a complete and positive answer to the conjecture by Diekmann and Heesterbeek [9, page 218].

System (5.2) always has the trivial equilibrium $P_{0}(0,0)$. The isoclines at which $\dot{y}=0$ are $y=0$ and $\gamma z=\gamma-\alpha-b+(b-\gamma+\alpha(1-f)) y$, which is a straight line intersecting the line $y=0$ at $z=z_{1}=1-(\alpha+b) / \gamma$ and the line $y=1$ at $z=z_{2}=-\alpha f / \gamma$. Note that $z_{1}<1$ and $z_{1}>0$ if and only if (5.1) is satisfied, while $z_{2} \leq 0$ always. If $0<f \leq 1$ then the isoclines at which $\dot{z}=0$ are given by

$$
z=\frac{f \alpha y}{b-b y-(1-f) \alpha y}
$$




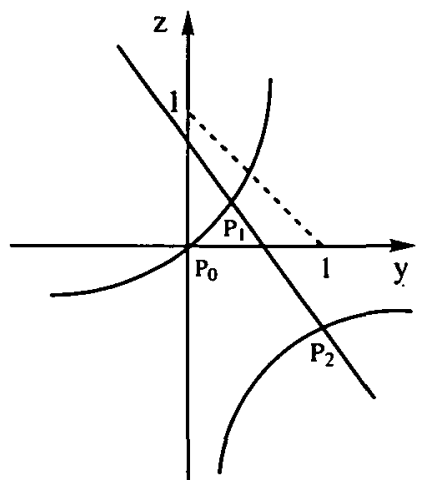

(a)

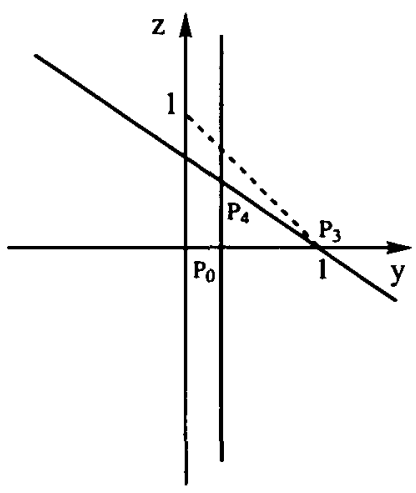

(b)

FIGURE 1. The equilibria for system (5.2) under various conditions: (a) $0<f \leq 1$; (b) $f=0$.

which are two increasing functions of $y$ (these two isoclines have vertical asymptote $y=y_{1}=b /(b+(1-f) \alpha)$ and horizontal asymptote $z=z_{3}=-f \alpha /(b+(1-f) \alpha)$ respectively). So there are two intersections with the sloping isocline

$$
\gamma z=\gamma-\alpha-b+(b-\gamma+\alpha(1-f)) y
$$

whenever (5.1) is satisfied. One equilibrium $P_{1}$ is in the interior of the triangle $D$ and another equilibrium $P_{2}$ is in the region $\left\{(y, z): y>y_{1}, z<z_{3}\right\}$. If $f=0$ then the isoclines at which $\dot{z}=0$ are $z=0$ and $y=b /(b+\alpha)$. Thus the system (5.2) has an equilibrium $P_{0}(0,0)$, an equilibrium $P_{3}(1,0)$, and an equilibrium $P_{4}$ in the interior of the triangle $D$. The equilibria of system (5.2) are illustrated in Figure 1 under various conditions.

The variational matrix of system (5.2) is given by

$$
J(y, z)=\left(\begin{array}{cc}
\gamma(1-z)-\alpha-b+2 y[\alpha(1-f)+b-\gamma] & -\gamma y \\
f \alpha+(1-f) \alpha z+b z & (1-f) \alpha y-b(1-y)
\end{array}\right) .
$$

The stability of the equilibria $P_{0}, P_{1}, P_{2}, P_{3}$ and $P_{4}$ is determined by the eigenvalues of the matrices $J\left(P_{0}\right), J\left(P_{1}\right), J\left(P_{2}\right), J\left(P_{3}\right)$ and $J\left(P_{4}\right)$ respectively.

LEMMA 5.2. If $0<f \leq 1$ and (5.1) is satisfied, then

(i) the equilibrium $P_{1}$ is locally asymptotically stable;

(ii) the equilibria $P_{0}$ and $P_{2}$ are saddle points.

PROOF. (i) See Diekmann and Heesterbeek [9, page 218]. 
(ii) The variational matrix of the system (5.2) at $P_{2}$ is

$$
J\left(P_{2}\right)=\left(\begin{array}{cc}
y(-\gamma+\alpha(1-f)+b) & -\gamma y \\
f \alpha+(1-f) \alpha z+b z & (1-f) \alpha y-b(1-y)
\end{array}\right) .
$$

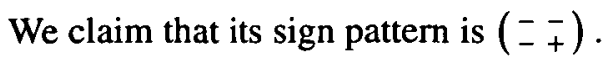

The signs of the anti-diagonal elements are clear, so we concentrate on the diagonal elements, starting with position 11. The assumption (5.1) implies $\gamma>b+\alpha$, or $-\gamma+b+\alpha<0$, and hence centainly $-\gamma+b+\alpha-\alpha f<0$. The element at position 22 is positive for large $y$, but changes sign at $y=b /(b+(1-f) \alpha)$. Hence the determinant of $J\left(P_{2}\right)$ is negative and $P_{2}$ is a saddle point.

It is easy to see that the variational matrix of the system (5.2) at $P_{0}$ is

$$
J\left(P_{0}\right)=\left(\begin{array}{cc}
\gamma-\alpha-b & 0 \\
f \alpha & -b
\end{array}\right) .
$$

The assumption (5.1) implies that its sign pattern is $\left(\begin{array}{c}+0 \\ +-\end{array}\right)$.

So the determinant of $J\left(P_{0}\right)$ is negative and $P_{0}$ is also a saddle point. The proof of Lemma 5.2 is now complete.

In quite the same manner, we can prove the following result.

LEMMA 5.3. If $f=0$ and (5.1) is satisfied, then

(i) the equilibrium $P_{4}$ is locally asymptotically stable;

(ii) the equilibria $P_{0}$ and $P_{3}$ are saddle points.

PROOF OF THEOREM 5.1. Because the $z$-axis is invariant, at the $y$-axis we have $d z / d t=f \alpha y \geq 0$, and on the line $y+z=1$ we have $d(y+z) / d t=b(y-1)<0$ for $y<1$. Hence $D$ is positively invariant, and in particular, every positive semiorbit starting in $D$ is bounded. By Lemmas $5.2-5.3$, the point $(\bar{y}, \bar{z})$ is locally asymptotically stable. The Poincaré-Bendixson theorem implies that the $\omega$-limit set $\omega\left(\gamma_{(1.2)}^{+}\right)$is therefore either the point $(\bar{y}, \bar{z})$ or a nontrivial periodic orbit. Therefore the proof of the conjecture is completed by showing that system (5.2) has no nontrivial periodic orbit in $D$.

Define $B(y, z)=y^{-1} z^{-1}$ for $(y, z) \in D$. By $(5.1)$, we have

$$
\begin{aligned}
\frac{\partial\left(B F_{1}\right)}{\partial y}+\frac{\partial\left(B F_{2}\right)}{\partial z}= & y^{-1} z^{-1}((\alpha+b-\gamma-\alpha f) y-\gamma z \\
& +(\gamma-\alpha-b)+(\alpha+b-\gamma-\alpha f) y) \\
& -y^{-1} z^{-1}((\alpha+b-\gamma-\alpha f) y-\gamma z+(\gamma-\alpha-b)) \\
& +y^{-1} z^{-1}((b+\alpha(1-f)) y-b)
\end{aligned}
$$




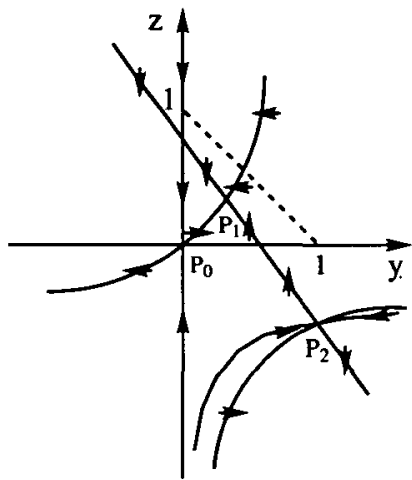

(a)

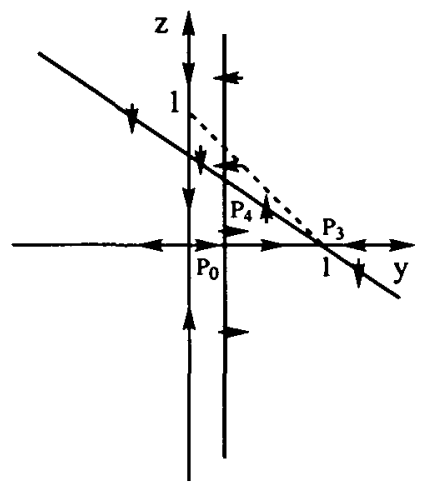

(b)

FIGURE 2. The direction field chart for system (5.2) under various conditions: (a) $0<f \leq 1$; (b) $f=0$.

$$
\begin{aligned}
& -y^{-1} z^{-2}(f \alpha y+(b+\alpha(1-f)) y z-b z) \\
= & -y^{-1} z^{-1}\left((\gamma+\alpha f-\alpha-b) y+f \alpha y z^{-1}\right)<0
\end{aligned}
$$

for all $(y, z) \in D$.

Thus the conditions of the Bendixson-Dulac theorem are satisfied and system (5.2) has no nontrivial periodic orbit in $D$. Therefore by the Poincaré-Bendixson theorem, the point $(\bar{y}, \bar{z})$ is globally asymptotically stable in $D$. The proof of Theorem 5.1 is now complete.

Since

$$
\frac{d z}{d y} \rightarrow \frac{b-(b+(1-f) \alpha) y}{\gamma y} \text { as } z \rightarrow \infty, y>0,
$$

it is easy to see that system (5.2) has no vertical asymptote in the right half-plane $(y>0)$. Moreover, we have the following theorem.

THEOREM 5.4. (1) For the case $f=0$, if (5.1) is satisfied, then

$$
\lim _{t \rightarrow \infty}(y(t), z(t))=(\bar{y}, \bar{z})
$$

if and only if $y(0)>0$ and $z(0)>0$.

(2) For the case $0<f \leq 1$, if (5.1) is satisfied, then $\lim _{t \rightarrow \infty}(y(t), z(t))=(\bar{y}, \bar{z})$ if and only if $(y(0), z(0)) \in G_{1}$, where $G_{1}$ is the region on the right half-plane and above $\Gamma$, where $\Gamma$ is the stable manifold of saddle point $P_{2}$. 
Proof. Since (5.3) holds, it is easy to see that system (5.1) has no vertical asymptote in the right half-plane $(y>0)$. For the case (b): $f=0$, from Figure 2 (the direction field chart for system (5.1)) and the above results we see that system (5.1) cannot have positive periodic solutions. It follows from the phase plane analysis that $\lim _{t \rightarrow \infty}(y(t), z(t))=(\bar{y}, \bar{z})$ if and only if $y(0)>0$ and $z(0)>0$. For the case (a): $0<f \leq 1$, similarly. From Figure 2 and the phase plane analysis it is easy to verify that $\lim _{t \rightarrow \infty}(y(t), z(t))=(\bar{y}, \bar{z})$ if and only if $(y(0), z(0)) \in G_{1}$.

\section{Summary}

In this paper, we extend the SIR epidemic models (1.2) in Diekmann and Heesterbeek [9] into SEIR type (1.3) with a constant exposed period. Our model is different from previous delayed epidemiological models in which the delay-dependent coefficients $e^{-\mu \tau}$ is ignored $([2,3,25,29,30])$ and where only the infective term $I(t)$ of the incidence term $\gamma S(t) l(t) / N(t)$ has delay $([2,3,25])$. Our model is also different from the delayed SEIR model with delay-dependent coefficients by Cooke et al. [8], as in our model we assume infected individuals lose the ability to give birth and when an individual is removed from the $I$-class, it recovers and acquires permanent immunity with probability $f(0 \leq f \leq 1)$ and dies from the disease with probability $1-f$.

By constructing a proper Lyapunov function, we get that (in Theorem 3.1) the disease-free equilibrium $(1,0,0,0)$ is globally attracting provided $\gamma /(b+\alpha)<1$. Using Thieme's persistence criteria [27] for infinite-dimensional systems, we prove in Theorem 4.1 that the system will be endemic in the sense of permanence when $R_{0}=$ $\gamma e^{-b \tau} /(b+\alpha)>1$. Since $R_{0}$ involves the latent delay $\tau$, as $\tau$ increases gradually, $R_{0}$ will get smaller and smaller and consequently the condition for permanence will become less likely to be satisfied. This suggests that the longer the exposed period the system has, the less the chances are that it will be endemic, that is, it is helpful for us to get the disease-free property by properly enlarging the exposed period.

Our disease-free result, Theorem 3.1, generalises the corresponding results by Diekmann and Heesterbeek [9] for system (1.2). Further, in Theorem 5.1, our results show that for system (1.2), existence of the endemic equilibrium indicates its global stability, which completely answers the conjecture proposed by Diekmann and Heesterbeek [9].

We believe $R_{0}=\gamma e^{-b \tau} /(b+\alpha)$ is an important threshold parameter for the diseasefree and endemic cases, however, in this paper, it still remains unsolved for

$$
1<\frac{\gamma}{b+\alpha} \text { and } \frac{\gamma}{b+\alpha} e^{-b \tau}<1
$$

We conjecture it will lead to the disease-free property. We leave this problem for our future work. 


\section{Acknowledgements}

The authors would like to express their thanks to the anonymous referee for his careful reading, to Professor Mats Gyllenberg for his valuable comments, and to Dr. Yanni Xiao and Prof. Wanbiao Ma for sending us some important references. The work of S. Q. Liu was partially performed while he was visiting the Institute of Biomathematics at the University of Urbino in Italy during April-May 2005. The financial support of these institutions is acknowledged. S. Q. Liu was also supported by the Chinese Postdoctoral Science Foundation and the National Natural Science Foundation of China (under Grant No. 10371127). The work of P. Yan was supported by the Academy of Finland and the National Natural Science Foundation of China (under Grant No. 10471119).

\section{References}

[1] M. E. Alexander and S. M. Moghadas, "Periodicity in an epidemic model with a generalized non-linear incidence", Math. Biosc. 189 (2004) 75-96.

[2] E. Beretta, T. Hara, W. B. Ma and Y. Takeuchi, "Global asymptotic stability of an SIR epidemic model with distributed time delay", Nonlinear Anal. 47 (2001) 4107-4115.

[3] E. Beretta and Y. Takeuchi, "Global stability of an sir epidemic model with time delays", J. Math. Biol. 33 (1995) 250-260.

[4] F. Brauer, "Models for the spread of universally fatal diseases", J. Math. Biol. 28 (1990) 451-462.

[5] F. Brauer, "Models for the spread of universally fatal diseases, II", in Differential Equation Models in Biology, Epidemiology and Ecology (eds. S. Busenberg and M. Martelli), Lecture Notes in Biomath. 92, (Springer, New York, 1991) 57-69.

[6] S. Busenberg and K. L. Cooke, Vertically transmitted diseases, Biomathematics 23 (SpringerVerlag, Berlin, 1993).

[7] S. Busenberg, K. L. Cooke and A. Pozio, "Analysis of a model of a vertically transmitted disease", J. Math. Biol. 17 (1983) 305-329.

[8] K. Cooke and P. Driessche, "Analysis of an SEIRS epidemic model with two delays", J. Math. Biol. 35 (1996) 240-260.

[9] O. Diekmann and J. A. P. Heesterbeek, Mathematical Epidemiology of Infectious Diseases (John Wiley \& Sons, Chichester, 2000).

[10] H. I. Freedman and P. Moson, "Persistence definitions and their connections", Proc. Amer. Math. Soc. 109 (1990) 1025-1033.

[11] L. Q. Gao and H. W. Herbert, "Disease transmission models with density-dependent demographics", J. Math. Biol. 30 (1992) 717-731.

[12] S. A. Gourley and Y. Kuang, "A stage structured predator-prey model and its dependence on through-stage delay and death rate", J. Math. Biol. 49 (2004) 188-200.

[13] J. K. Hale and P. Waltman, "Persistence in infinite-dimensional systems", SIAM J. Math. Anal. 20 (1989) 388-395.

[14] H. W. Hethcote, "Qualitative analyses of communicable disease models", Math. Biosci. 28 (1976) 335-356. 
[15] H. W. Hethcote, "Three basic epidemiological models", in Applied Mathematical Ecology (eds. L. Gross, T. G. Hallam and S. A. Levin), (Springer, Berlin, 1989) 119-144.

[16] H. W. Hethcote, “The mathematics of infectious diseases", SIAM Rev. 42 (2000) 599-653.

[17] H. W. Hethcote and P. Driessche, "An SIS epidemic model with variable population size and a delay", J. Math. Biol. 34 (1995) 177-194.

[18] H. W. Hethcote and P. Driessche, "Two SIS epidemiologic models with delays", J. Math. Biol. 40 (2000) 3-26.

[19] H. W. Hethcote, H. W. Stech and P. V. D. Driessche, "Nonlinear oscilllations in epidemic models", SIAM J. Appl. Math. 40 (1981) 1-9.

[20] W. O. Kermack and A. G. McKendrick, "Contributions to the mathematical theory of epidemics", Proc. Roy. Soc. A 115 (1927) 700-721.

[21] Y. Kuang, Delay Differential Equations with Applications in Population Dynamics (Academic Press, Boston, 1993).

[22] S. Liu, L. Chen, G. Luo and Y. Jiang, "Asymptotic behavior of competitive Lotka-Volterra system with stage structure", J. Math. Anal. Appl. 271 (2002) 124-138.

[23] S. Q. Liu and E. Beretta, "A stage-structured predator-prey model of Beddington-DeAngelis type", SIAM J. Appl. Math. 66 (2006) 1101-1129.

[24] S. Q. Liu and Z. J. Liu, "Permanence of general stage-structured consumer-resource models", J. Comp. Appl. Math. (in press).

[25] W. B. Ma, Y. Takeuchi, T. Hara and E. Beretta, "Permanence of an SIR epidemic model with distributed time delays", Tohoku Math. J. 54 (2002) 581-591.

[26] J. Mena-Lorca and H. W. Hethcote, "Dynamic models of infectious disease as regulators of population size", J. Math. Biol. 30 (1992) 693-716.

[27] H. R. Thieme, "Persistence under relaxed point-dissipativity (with application to an endemic model)", SIAM J. Math. Anal. 24 (1993) 407-435.

[28] H. R. Thieme, "Uniform persistence and permanence for non-autonomous semiflows in population biology”, Math. Biosc. 166 (2000) 173-201.

[29] W. D. Wang, "Global behavior of an SEIRS epidemic model with time delays", Appl. Math. Let. 15 (2002) 423-428.

[30] W. D. Wang and Z. E. Ma, "Global dynamics of an epidemic model with time delay", Nonlinear Analysis: Real World Applications 3 (2002) 365-373.

[31] Y. N. Xiao and L. S. Chen, "Modeling and analysis of a predator-prey model with disease in the prey”, Math. Biosc. 171 (2001) 59-82. 